\title{
Hypernatraemia in an adult in-patient population
}

\author{
C.A. Long, P. Marin*, A.J. Bayer, H.G.M. Shetty and M.S.J. Pathy \\ Department of Geriatric Medicine, Cardiff Royal Infirmary (West Wing), Newport Road, \\ Cardiff CF2 1SZ, UK.
}

\begin{abstract}
Summary: We report a retrospective study of hypernatraemia (serum sodium concentration $>150$ mmol/l) in an adult in-patient population of a health district during one year. The incidence was $0.3 \%$ with at least $60 \%$ of cases developing after hospital admission, mainly in elderly patients. Dehydration appeared to be the major cause, with the use of diuretics, depressed conscious level or febrile illness implicated in a majority. Most patients had more than one contributory factor and iatrogenic causes were common. Associated illnesses were often severe and the in-hospital mortality was high (54\%) regardless of age. Hypernatraemia in hospitalized patients should be largely avoidable and there is a need for greater awareness of the importance of active maintenance of hydration in susceptible patients.
\end{abstract}

\section{Introduction}

Reports of hypernatraemia in the American literature ${ }^{1,2,5}$ emphasize that it is an important condition to recognize. It carries a high mortality rate (up to $70 \%)^{1,2}$ and yet should be a largely avoidable condition because it most commonly results from dehydration and iatrogenic factors. ${ }^{1,2}$ Elderly people ${ }^{3,4}$ and other dependent groups are particularly at risk and it has indeed been suggested that hypernatraemia dehydration might be an indicator of neglect in private nursing homes. ${ }^{2}$

In contrast, there are few reports of the incidence and general characteristics of hypernatraemia in British hospital practice. The most recent reports are confined to severe cases $^{6}$ (serum sodium in excess of $160 \mathrm{mmol} / \mathrm{l}$ ) and the occurrence of hypernatraemia in mentally handicapped patients. ${ }^{7}$ The paucity of data regarding the incidence, aetiology and outcome of hypernatraemia, particularly in relation to the elderly, suggests the need for a more comprehensive study.

We report a retrospective study of hypernatraemia (serum sodium concentration $>150 \mathrm{mmol} / \mathrm{l}$ ) among the adult in-patient population of a health district during one year.

\section{Methods}

The study population comprised in-patients over 18 years of age and was derived from all the acute

*Present address: Department of Care of the Elderly, Watford General Hospital, Vicarage Road, Watford, Herts WD1 8HB, UK.

Correspondence: C.A. Long, M.R.C.P. (UK)

Accepted: 11 February 1991 general, rehabilitation and continuing care hospitals of South Glamorgan Health Authority.

The definition of hypernatraemia is arbitrary, but Ross and Christie ${ }^{8}$ in their comprehensive review suggest that most authors define hypernatraemia as a serum sodium concentration $>150$ $\mathrm{mmol} / \mathrm{l}$ and this convention has been used in the study. Instances of hypernatraemia during 1987 were identified by a computer and hand search of all serum sodium estimations performed by the four biochemistry departments serving the hospitals. The study was retrospective and so the reasons sodium estimations were requested could not always be determined. Ion specific electrodes (Beckman Astra) were used to estimate serum sodium and the reference range for the laboratories was 135 to $143 \mathrm{mmol} / \mathrm{l}$.

Relevant clinical information was obtained from a review of the patients' medical case notes and the patients' present status confirmed, where necessary, by contacting the general practitioner. Statistical analysis was by chi-squared tests, using the Statistical Package for the Social Sciences (SPSSX).

\section{Results}

Of the 236 hypernatraemia patients initially identified, 13 were aged under 18 years and 17 were out-patients. There were thus 206 adult in-patients with hypernatraemia, out of the approximately 75,000 non-paediatric admissions during the year, yielding an overall incidence of 0.3 per cent. None were from continuing care hospitals for the elderly mentally or physically infirm or for the mentally 
handicapped.

The case notes of these 206 patients were then sought and $160(78 \%)$ were located. These appeared to be representative of the group as a whole. The median age of the patients was 76 years, ranging from 18 to 98 years. There were $82(51 \%)$ men and $78(49 \%)$ women. There were 35 patients living in residential or nursing homes, though at least 19 of these were normonatraemic at the time of hospital admission.

Eighty-seven (54\%) of the patients were on intensive therapy units when hypernatraemia was identified and the other cases were on medical, surgical and geriatric wards. Hypernatraemia was noted within 24 hours of admission in $51(32 \%)$ patients, whereas $96(60 \%)$ patients were initially documented as normonatraemic with a raised serum sodium developing during their hospital stay. In $13(8 \%)$ of the patients there was a delay in electrolyte estimation and the time and place of onset of hypernatraemia was uncertain.

The possible underlying causes of hypernatraemia are shown in Table I.

Dehydration appeared to be a major factor in most patients. Many had more than one contributory cause, with the use of diuretics, depressed conscious level and febrile illness each being implicated in more than half of the study patients. There were some significant differences between the young and elderly. Self neglect was a finding in exclusively older patients and in whom there was a greater incidence of diabetes mellitus. The younger age group suffered more febrile illnesses and had recently undergone more surgical procedures and other therapeutic interventions, including artificial ventilation, dialysis and administration of solutes.
Underlying renal disease was more frequently present in the younger patients than the old [17 $(38 \%)$ vs $24(21 \%) ; P<0.05$ ], whereas the old were more likely to have underlying cardiovascular [89 (77\%) vs $21(47 \%) ; P<0.0001]$ and central nervous system disease [65 (57\%) vs $13 \quad(29 \%)$; $P<0.001]$.

Hypernatraemia was sustained for at least 48 hours in $66(41 \%)$ patients and persisted for up to 30 days in one patient. Rapid correction (within 48 hours) was achieved in only $35(22 \%)$ patients. Mortality was high; $45(28 \%)$ patients died before their hypernatraemia had been corrected and subsequently a further $41(26 \%)$ patients died before hospital discharge. Of the $74(46 \%)$ patients who went home, at least 18 patients died during the follow-up period of between 1 and 18 months. Age had no influence on the duration of hypernatraemia or in-hospital mortality.

\section{Discussion}

Hypernatraemia is an infrequent but serious complication, developing in the majority of patients, during the hospital stay. Over half the patients were being managed on intensive therapy units, suggesting underlying severe and life threatening illness. The estimated overall incidence of hypernatraemia of $0.3 \%$ compares favourably with the previouslyo published estimate 1 of $1.1 \%$ among elderly patients in a general hospital in America. This study, however, used a less strict definition of hypernatraemia, with a cut-off at a serum sodium concentration of $149 \mathrm{mmol} / \mathrm{l}$.

The greater frequency of hypernatraemia among

Table I Possible contributory causes for hypernatraemia in 160 adult hospital in-patients with serum sodium $>150 \mathrm{mmol} / \mathrm{l}$, by age group. Most patients had more than one cause

\begin{tabular}{lrlrrr}
\hline & \multicolumn{3}{c}{$\begin{array}{c}\text { Number (\%) } \\
\text { Age patients } \\
(n=115)\end{array}$} & $\begin{array}{c}\text { Age }<65 \text { years } \\
(n=45)\end{array}$ & P value \\
\hline Diuretics & 85 & $(74)$ & 30 & $(67)$ & NS \\
Depressed sensorium & 81 & $(70)$ & 32 & $(71)$ & NS \\
Febrile illness & 64 & $(56)$ & 32 & $(71)$ & $<0.05$ \\
Diabetes mellitus & 23 & $(20)$ & 4 & $(9)$ & $<0.01$ \\
Administration solute & 21 & $(18)$ & 16 & $(35)$ & $<0.01$ \\
Ventilation & 21 & $(18)$ & 23 & $(51)$ & $<0.0001$ \\
Surgery & 16 & $(14)$ & 17 & $(38)$ & $<0.01$ \\
Gastrointestinal bleed & 15 & $(13)$ & 5 & $(11)$ & NS \\
Diarrhoea & 12 & $(10)$ & 5 & $(11)$ & NS \\
Self neglect & 8 & $(7)$ & & 0 & $<0.05$ \\
Parenteral nutrition & 5 & $(4)$ & 1 & $(2)$ & NS \\
Dialysis & 5 & $(4)$ & 8 & $(18)$ & $<0.01$ \\
Diabetes insipidus & 1 & $(1)$ & 2 & $(4)$ & NS \\
Other & 7 & $(6)$ & 6 & $(13)$ & NS \\
\hline
\end{tabular}

NS $=$ not significant 
elderly patients reflects both greater incidence of disease and diminished homeostatic capacity in old age. ${ }^{3,4}$ The outcome, however, was similar in young and elderly, with the high in-hospital mortality of $54 \%$ being comparable to the previously reported rates of between $42 \%{ }^{1}$ and $70 \%{ }^{2}$ In our study, it was difficult to ascertain how much of the excess mortality was due to the fluid and electrolyte disturbance and how much to the severity of underlying medical illness. Certainly, nearly half of those who succumbed had returned to normonatraemia prior to their death.

Many of the cases appeared to be related to the development of dehydration, often after hospital admission. Susceptible patients could be identified with a predictable risk, particularly those who were unable to obtain oral fluids themselves independently and those with excessive fluid loss, for example associated with fever or diarrhoea.

The clinical recognition of dehydration can be particularly difficult in the elderly, in whom failure to report thirst and the unreliability of physical

\section{References}

1. Snyder, N.A., Feigal, D.W. \& Arieff, A.I. Hypernatraemia in elderly patients. A heterogeneous, morbid and iatrogenic entity. Ann Intern Med 1987, 107: 309-319.

2. Himmelstein, D.U., Jones, A.A. \& Woolhandler, S. Hypernatraemic dehydration in nursing home patients. An indicator of neglect. J Am Geriatr Soc 1983, 31: 466-471.

3. Beck, L.H. \& Lavizzo-Mourey, R. Geriatric hypernatraemia. Ann Intern Med 1987, 107: 768-769.

4. Leaf, A. Dehydration in the elderly. $N$ Engl J Med 1984, 311: 791-792. signs such as laxity of the skin present special problems and requires carers to be constantly vigilant. It is noteworthy that no cases occurred among vulnerable patients on mental handicap, psychogeriatric or geriatric rehabilitation or continuing care wards.

Whilst the development of hypernatraemia seems highly associated with severe underlying medical illness, it is largely an avoidable complication. There seems to be a need for greater awareness amongst medical and nursing staff, particularly on acute wards, of the importance of active maintenance of hydration in susceptible patients.

\section{Acknowledgements}

We are grateful to the consultant staff of South Glamorgan Health Authority who granted us access to patient records and to Mrs Gwyneth Garner for her efficiency and determination in locating them.

5. Sadat, A., Paulman, P.M. \& Mathews, M. Hypernatraemia in the elderly. Am Fam Physician 1989, 40; 125-128.

6. Bhatnagar, D. \& Weinkove, C. Serious hypernatraemia in a hospital population. Postgrad Med J 1988, 64: 441-443.

7. Macdonald, N.J., McConnell, K.N., Stephen, M.R. \& Dunnigan, M.G. Hypernatraemic dehydration in patients in a large hospital for the mentally handicapped. $\operatorname{Br} \operatorname{Med} J 1989,299$ : 1426-1429.

8. Ross, E.J. \& Christie, S.B.M. Hypernatraemia. Medicine 1969, 48: 441-473. 